\title{
ZF-DFE TRANSCEIVER DESIGN FOR TIME-VARYING MIMO CHANNELS USING SPACE-TIME GENERALIZED TRIANGULAR DECOMPOSITION
}

\author{
Chih-Hao Liu, P. P. Vaidyanathan
}

\author{
Dept. of Electrical Engineering, MC 136-93 \\ California Institute of Technology, Pasadena, CA 91125, USA \\ E-mail: chliu@caltech.edu and ppvnath@systems.caltech.edu
}

\begin{abstract}
We consider the design of MIMO transceivers with zeroforcing $(\mathrm{ZF})$ decision feedback detection over time-varying MIMO channels. The data vectors are grouped into spacetime blocks (ST-blocks) for the spatial and temporal precoding to take advantage of the diversity offered by time-varying channels. We extend the generalized triangular decomposition (GTD) for the case of time-varying channels by introducing the space-time GTD (ST-GTD). Based on ST-GTD and the channel prediction, we propose the space-time geometric mean decomposition (ST-GMD) based system which minimizes the arithmetic mean square error (MSE) for every ST-block. We also present the causal ST-GTD based system which does not require channel prediction. The simulations show that this system achieves the same BER performance asymptotically as the ST-GMD based system. In moderate high SNR, the proposed systems have superior BER performance over the conventional GMD-based systems.
\end{abstract}

Index Terms- Generalized Triangular Decomposition, Transceivers, Time-Varying Channels, Space-time GTD.

\section{INTRODUCTION}

The introduction of multiple antennas which increases the channel capacity [4] offers an economical solution to meet the growing demand of broadband wireless communications. In the mobile IEEE 802.16e standard [3], the wireless channels are time-varying which is caused by Doppler shift due to users' mobility. In this paper, we consider the block fading model in which the channel is constant over the coherence (block) interval of $T_{c}$ symbol vectors. The channel varies across different coherence intervals independently or according to Jakes' model [4], [2].

To exploit the array gain for the full channel capacity, both channel state information (CSI) at the transmitter and the receiver are required [4]. Based on the CSI at both ends, we consider the optimal MIMO transceiver design under zeroforcing constraint. For the time invariant channels, the GMDbased system [6], [7], is shown to minimize the arithmetic mean (over the spatial domain) of the expected MSE at the input of the decision device and the average BER in high SNR [7]. Moreover, it is also shown to achieve optimal channel throughput asymptotically in high SNR [6].

In the case of time-varying channels, if the GMD-based system is directly applied, the average BER is not minimized since different coherence blocks have different arithmetic MSEs at the feedback loop detector. Based on the GTD [8],

Work supported in parts by the ONR grant N00014-08-1-0709 and the California Institute of Technology. we develop space-time or ST-GTD for the decomposition of time-varying MIMO channels. Using the special case of ST-GTD, namely ST-GMD which does GMD on both spatial and temporal domains, we develop the ST-GMD transceiver. It jointly minimizes the arithmetic MSE in each ST-block (which consists of several coherence blocks), and minimizes the average BER in high SNR. The transmitter (TX) of the ST-GMD transceiver requires perfect channel prediction. Similar issues have been addressed in several papers, i.e., [1], [2]. The Wiener filter theory is usually adopted for the prediction of future channel coefficients based on the previous channel estimations.

A causal ST-GTD based transceiver (CST-GTD) with stationary temporal processing is also proposed here. It does not require channel prediction because its temporal precoder is constant. It is shown that the CST-GTD has smaller arithmetic MSE than the GMD-based system. The simulation also shows that the BER performance of CST-GTD approximates that of the ST-GMD transceiver asymptotically as the size of a ST-block increases.

Notations: $(\cdot)^{T}$ and $(\cdot)^{\dagger}$ denote the transpose and the conjugate transpose, respectively. $x_{i}$ or $[\mathbf{x}]_{i}$ denotes the $i$ th element of a vector $\mathbf{x} ; A_{i, j}$ or $[\mathbf{A}]_{i, j}$ denotes the $(i, j)$ th element of a matrix A. $\mathbf{I}_{M}$ denotes the $M \times M$ identity matrix. $E(\cdot)$ stands for expectation. $\operatorname{diag}(\mathbf{x})$ is a diagonal matrix with the entries of $\mathbf{x}$ on the diagonal. $\mathbf{u} \prec+\mathbf{v}$ and $\mathbf{u} \prec x \mathbf{v}$ denote additive and multiplicative majorization, respectively [5], [8].

\section{PROBLEM FORMULATION AND REVIEW}

In this paper, we consider the narrowband block fading MIMO channel model [4]. The channel remains constant over the coherence period of $T_{c}$ symbol vectors and varies independently [4] or according to Jakes' model [2] across different coherence intervals. One data block which consists of $T_{c}$ symbol vectors is transmitted during each coherence (block) interval. For the simplicity of analysis, we just pick one symbol vector from each block since the symbol vectors in the same block go through the same channel. The channel model is given by

$$
\mathbf{y}(k)=\mathbf{H}(k) \mathbf{a}(k)+\mathbf{w}(k)
$$

where $k$ is the block index, $\mathbf{H}(k)$ is a $J \times M$ full rank channel matrix with $J \geq M$. a $(k)$ is an $M \times 1$ symbol vector from the $k$ th block with each element $[\mathbf{a}(k)]_{i}$ chosen from the alphabet $\chi$ of finite size. The noise $\mathbf{w}(k)$ is a $J \times 1$ Gaussian random process vector with $E(\mathbf{w}(k))=\mathbf{0}$ and $E\left(\mathbf{w}(k) \mathbf{w}^{\dagger}\left(k^{\prime}\right)\right)=$ $\sigma_{w}^{2} \delta\left(k-k^{\prime}\right) \mathbf{I}_{J}$. And $\mathbf{y}(k)$ is the $J \times 1$ received vector. At each coherence interval, $\mathbf{H}(k)$ is assumed to be known to the 
transmitter and receiver. There are standard methods to estimate this [2], [6]. In the following, a brief review of GTD theorem is given.

Theorem 1: The generalized triangular decomposition [8]: Let $\mathbf{H} \in \mathbb{C}^{J \times N}$ have rank $M$ with non-zero singular values $\mathbf{d}_{H}=\left[\sigma_{H, 0}, \sigma_{H, 1}, \cdots, \sigma_{H, M-1}\right]^{T}$, then there exists an upper triangular matrix $\mathbf{R} \in \mathbb{C}^{M \times M}$, and semi-unitary matrices $\mathbf{P} \in \mathbb{C}^{N \times M}$ and $\mathbf{Q} \in \mathbb{C}^{J \times M}$ in which all columns are orthonormal, such that $\mathbf{H}=\mathbf{Q R P}^{\dagger}$ if and only if $\overline{\mathbf{r}} \prec \times \mathbf{d}_{H}$ where $\bar{r}_{i}=\left|R_{i i}\right|$.

Suppose $R_{i i}=\left|R_{i i}\right| e^{j \theta_{i}}$. Without loss of generality, $R_{i i}$ can be made real and positive by multiplying the $i$ th column of $\mathbf{Q}$ by $e^{j \theta_{i}}$. By restricting $R_{i i}$ to be equal to the geometric mean of the $M$ non-zero singular values of $\mathbf{H}$, GTD reduces to GMD [6].

\section{SPACE-TIME GTD}

To facilitate space-time processing, $K$ blocks of symbol vectors are grouped into one space-time block as

$$
\mathbf{a}_{m}=\left[\begin{array}{lll}
\mathbf{a}^{T}(m K) & \ldots & \mathbf{a}^{T}(m K+K-1)
\end{array}\right]^{T},
$$

and the ST-block index $m$ and $m K$ will be omitted for convenience. The equivalent MIMO channel matrix for the $m$ th ST-block is a $K J \times K M$ block diagonal matrix given by

$$
\mathbf{H}=\operatorname{diag}(\mathbf{H}(0), \mathbf{H}(1), \ldots, \mathbf{H}(K-1)) .
$$

Let $0 \leq k \leq K-1$. Suppose that GMD is applied to each $\mathbf{H}(k)$ separately (in spatial domain). We have $\mathbf{H}(k)=$ $\mathbf{Q}(k) \mathbf{R}(k) \mathbf{P}^{\dagger}(k)$, where $\mathbf{Q}(k) \in \mathbb{C}^{J \times M}$ and $\mathbf{P}(k) \in \mathbb{C}^{M \times M}$ are semi-unitary matrices and $\mathbf{R}(k) \in \mathbb{C}^{M \times M}$ is an upper triangular matrix with the diagonal vector $\mathbf{r}(k)$. The entries of $\mathbf{r}(k)$ are equal such that

$$
r_{i}(k)=\sigma_{k}=\left(\prod_{i=0}^{M-1} \sigma_{H, i}(k)\right)^{1 / M},
$$

where $\sigma_{H, i}(k)$ is the $i t h$ largest singular value of $\mathbf{H}(k)$. Now, $\mathbf{H}$ in (3) can be decomposed as

$$
\mathbf{H}=\mathbf{Q R P}^{\dagger},
$$

where $\mathbf{Q}, \mathbf{P}$ and $\mathbf{R}$ are block diagonal matrices with $\mathbf{Q}(k)$, $\mathbf{P}(k)$ and $\mathbf{R}(k)$ on the diagonals, respectively. Let $\mathbf{d}=$ $\left[\sigma_{0}, \ldots, \sigma_{K-1}\right]^{T} . \mathbf{R}$ can be expressed as

$$
\mathbf{R}=\mathbf{D}_{\mathbf{R}}\left(\mathbf{I}_{K M}+\mathbf{B}\right)
$$

where $\mathbf{D}_{\mathbf{R}}=\mathbf{\Sigma} \otimes \mathbf{I}_{M}, \boldsymbol{\Sigma}=\operatorname{diag}(\mathbf{d})$, and $\mathbf{B}$ is a block diagonal matrix with $\mathbf{B}(k)$ on the diagonal. $\mathbf{B}(k)$ are strictly upper triangular $M \times M$ matrices. Since $\boldsymbol{\Sigma}$ is a diagonal matrix consisting of positive entries, the diagonal elements $\sigma_{k}$ are also its singular values. Therefore, by theorem 1 , we can decompose $\boldsymbol{\Sigma}$ (in time domain) as

$$
\boldsymbol{\Sigma}=\mathbf{Q}_{1} \mathbf{R}_{1} \mathbf{P}_{1}^{\dagger}
$$

where $\mathbf{P}_{1}$ and $\mathbf{Q}_{1}$ are $K \times K$ unitary matrices, and $\mathbf{R}_{1}$ is a $K \times K$ upper triangular matrix. The necessary and sufficient condition for (7) to be possible is $\mathbf{r}_{1} \prec x \mathbf{d}$ in which $\mathbf{r}_{1}$ is a $K \times 1$ vector consisting of the diagonal elements of $\mathbf{R}_{1}$. By (5), (6) and (7), the full rank block diagonal matrix $\mathbf{H}$ in (3) can be decomposed as

$$
\mathbf{H}=\mathbf{Q}\left(\left(\mathbf{Q}_{1} \mathbf{R}_{1} \mathbf{P}_{1}^{\dagger} \otimes \mathbf{I}_{M}\right)\left(\mathbf{I}_{M K}+\mathbf{B}\right)\right) \mathbf{P}^{\dagger},
$$

if and only if $\mathbf{r}_{1} \prec_{\times} \mathbf{d}$. The decomposition taking this form is referred to as the ST-GTD. We denote

$$
\mathbf{D}_{\mathbf{R}_{1}}=\operatorname{diag}\left(\mathbf{r}_{1}\right), \mathbf{B}_{1}=\mathbf{D}_{\mathbf{R}_{1}}{ }^{-1} \mathbf{R}_{1}-\mathbf{I}_{K}
$$

When the entries of $\mathbf{r}_{1}$ equal $\left(\prod_{k=0}^{K-1} \sigma_{k}\right)^{1 / K}$, the time domain GTD in (7) reduces to the time domain GMD. We name this kind of ST-GTD, in particular, as ST-GMD.

\section{SPACE-TIME GTD TRANSCEIVERS}

In this section, we propose the ST-GTD ZF-DFE transceiver based on the ST-GTD introduced in the last section. The precoder of ST-GTD transceiver is shown in Fig. 1 and the receiver is in Fig. 2. Here, it is assumed that the transmitter could predict the channels $\mathbf{H}(k)$ for $0 \leq k \leq K-1$ perfectly before sending a ST-block $\mathbf{a}$. We also assume that the receiver can track the channels perfectly and the decoding follows after the reception of a whole ST-block.

Since the transmitter and receiver have perfect CSI of $\mathbf{H}$, ST-GTD can be applied to decompose $\mathbf{H}$ as (8) to get $\mathbf{P}, \mathbf{P}_{1}$, $\mathbf{Q}, \mathbf{Q}_{1}, \mathbf{B}$ and $\mathbf{B}_{1}$ for the precoder and the receiver. Note that $\mathbf{D}_{\mathbf{R}}$ should be chosen as $\operatorname{diag}(\mathbf{d}) \otimes \mathbf{I}_{M}$ and $\mathbf{D}_{\mathbf{R}_{1}}=\operatorname{diag}\left(\mathbf{r}_{1}\right)$ where $\mathbf{r}_{1} \prec_{x} \mathbf{d}$. At each ST-block time, a is precoded by the linear precoder, transmitted through the channel $\mathbf{H}$ and pre-processed at the receiver by $\mathbf{Q}^{\dagger}, \alpha^{-1} \mathbf{D}_{\mathbf{R}}{ }^{-1}$. The estimation of $\mathbf{a}$ is obtained by the following successive cancelation algorithm, which we describe after the introduction of some notations. For $0 \leq i \leq M-1$, define an $M \times M$ diagonal matrix $\mathbf{E}_{i}$ as

$$
\mathbf{E}_{i}=\operatorname{diag}([\underbrace{0, \ldots, 0}_{M-i}, \underbrace{1, \ldots, 1}_{i}]),
$$

and an $M \times 1$ vector $\mathbf{s}_{i}$ in which all the entries are zero except that the $i$ th entry is 1 . Based on $\mathbf{E}_{i}$ and $\mathbf{s}_{i}$, we define two operators, $\boldsymbol{\Theta}_{i}=\mathbf{I}_{K} \otimes \mathbf{E}_{i}$ and $\mathbf{S}_{i}=\mathbf{I}_{K} \otimes \mathbf{s}_{i}^{T}$, on a ST-block vector a which has the form as (2). $\boldsymbol{\Theta}_{i}$ retains the last $i$ symbols of each $\mathbf{a}(k)$ and makes the other symbols of $\mathbf{a}(k)$ zero. $\mathbf{S}_{i} \mathbf{a}=\left[a_{i}(0), \ldots, a_{i}(K-1)\right]^{T}$.

Successive cancelation detection algorithm:

1. Initialize: $i=0$.

2. Outer loop feedback (space domain): Calculate

$$
\mathbf{S}_{M-i-1} \mathbf{y}=\mathbf{S}_{M-i-1}\left(\mathbf{y}^{\prime}-\mathbf{B}\left(\mathbf{P}_{1} \otimes \mathbf{I}_{M}\right) \boldsymbol{\Theta}_{i} \hat{\mathbf{a}}\right) \text {. }
$$

3. Loop feedback and detection (time domain): Compute

$\mathbf{z}^{(i)}=\mathbf{S}_{M-i-1} \mathbf{r}^{\prime}=\left(\mathbf{I}_{K}+\mathbf{B}_{1}\right) \mathbf{P}_{1}^{\dagger} \mathbf{S}_{M-i-1} \mathbf{y} \cdot \mathbf{S}_{M-i-1} \hat{\mathbf{a}}$ can be decoded sequentially with the following procedures:

(a) $\hat{\mathbf{a}}((K-1) M+M-1-i)=Q t\left(z_{K-1}^{(i)}\right)$ where the function $q=Q t(t)$ sets $q$ to the element in $\chi$ such that it is closest to $t$ in Euclidean norm.

(b) For $c=2, \ldots, K$, $\hat{\mathbf{a}}((K-c) M+M-1-i)=$ $Q t\left(z_{K-c}^{(i)}-\sum_{m=K-c+1}^{K-1}\left[\mathbf{B}_{1}\right]_{K-c, m} \cdot \hat{\mathbf{a}}(m M+\right.$ $M-i-1))$. 


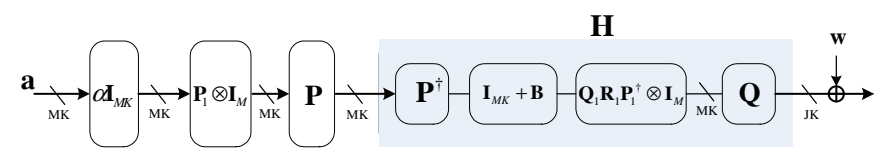

Fig. 1. The transmitter of the ST-GTD transceiver and the channel

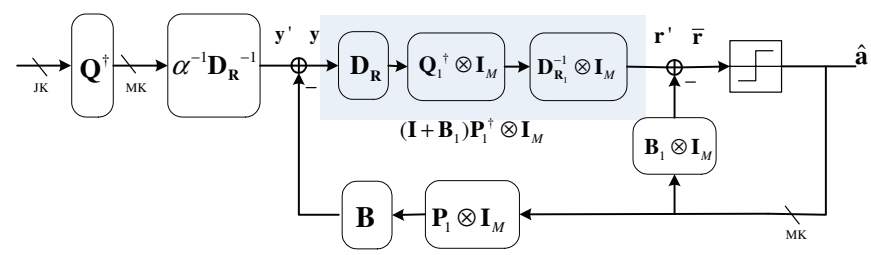

Fig. 2. The receiver of the ST-GTD transceiver

4. If $i=M-1$, then stop, else set $i=i+1$ and goto 2 .

A detailed justification of the algorithm can be found in [9].

The performance of the ST-GTD transceiver in Fig. 2 mainly depends on the noise component $\mathbf{e}$ in $\overline{\mathbf{r}}$. The error covariance matrix $\mathbf{R}_{\mathrm{ee}}$ of the noise component under no error propagation assumption is given by

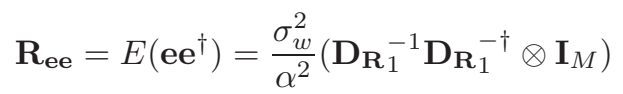

The total MSE of the ST-GTD transceiver over a ST-block is

$$
\xi_{s t-g t d}=\frac{\sigma_{w}^{2} M}{\alpha^{2}} \sum_{k=0}^{K-1} \frac{1}{\left|\eta_{k}\right|^{2}} \geq \frac{\sigma_{w}^{2} M K}{\alpha^{2}}\left(\prod_{k=0}^{K-1} \frac{1}{\sigma_{k}^{2}}\right)^{1 / K},
$$

where $\eta_{k}=\left[\mathbf{r}_{1}\right]_{k}$. The last inequality comes from AM-GM inequality and $\mathbf{r}_{1} \prec_{x} \mathbf{d}$. The equality holds when $\left|\eta_{0}\right|=$ $\ldots=\left|\eta_{K-1}\right|$ and $\mathbf{r}_{1} \prec \times \mathbf{d}$. In particular, if we choose

$$
\eta_{k}=\left(\prod_{k=0}^{K-1} \sigma_{k}\right)^{1 / K},
$$

then $\mathbf{r}_{1} \prec_{x} \mathbf{d}$ is also satisfied, making the ST-GTD possible. This is the case when ST-GMD is applied. We call this class of ST-GTD transceiver the ST-GMD transceiver. The total MSE of the ST-GMD transceiver is given by

$$
\xi_{s t-g m d}=\frac{\sigma_{w}^{2} M K}{\alpha^{2}}\left(\prod_{k=0}^{K-1} \frac{1}{\sigma_{k}^{2}}\right)^{1 / K}
$$

The class of ST-GMD transceivers is the optimal subclass of ST-GTD transceivers in terms of total MSE. Notice that the ST-GMD allows the ST-GMD transceiver to have the optimal MSE in (14), which is the smallest MSE achievable by directly applying GMD to the big matrix $\mathbf{H}$. Furthermore, the error covariance matrix of ST-GMD transceiver has equal diagonal elements. Hence, for every ST-block, the STGMD transceiver minimizes both the arithmetic and geometric MSE, and the average un-coded BER in moderate high SNR according to [7]. In addition, suppose that SVD of $\mathbf{H}$ is given, ST-GTD requires lower computational complexity, $O(K M(M+J))+O\left(K^{2}\right)$, than the complexity of directly applying GTD on $\mathbf{H}$, which is $O\left(K^{2} M(M+J)\right)$ [8]. Moreover, ST-GTD is more suitable for the design of ST-GTD transceivers without channel prediction which will be discussed in section 5 .

\section{SPACE-TIME GTD TRANSCEIVERS WITH STATIONARY TEMPORAL PROCESSING}

In this section, we develop the ST-GTD transceiver which does not rely on channel prediction. This means the transmitter is causal. Note that casuality is not an issue for the receiver, since the receiver can store the signal until the whole ST-block is received before it starts to decode. In section 4, the computation of precoding matrix $\mathbf{P}_{1}$ requires the knowledge of the singular values of $\mathbf{H}(0), \ldots, \mathbf{H}(K-1)$. At time $k=0$, the precoder already needs $\mathbf{P}_{1}$ to precode one block for transmission. To make the precoder causal, one can let $\mathbf{P}_{1}$ be a constant unitary matrix $\mathbf{W}$. In [10], the DFT or Hadamard matrix is chosen as the channel independent precoder for the OFDM system to equalize the MSEs over subchannels and hence minimize average BER. This motivates us to choose $\mathbf{W}$ to be a DFT or Hadamard matrix. The causal implementation of the spatial precoder $\mathbf{P}$ is not a problem, since $\mathbf{P}$ is block diagonal matrix consisting of $\mathbf{P}(k)$ and the computation of $\mathbf{P}(k)$ requires only the current CSI $\mathbf{H}(k)$. Letting $\mathbf{a}^{\prime}=\left(\mathbf{W} \otimes \mathbf{I}_{M}\right) \mathbf{a}$, the precoded block at time $k$ is given by $\mathbf{P}(k)\left[\mathbf{a}^{\prime}\right]_{k M: k M+M-1}$.

Originally, $\mathbf{Q}_{1}, \mathbf{P}_{1}$ and $\mathbf{R}_{1}$ are obtained from the time domain GTD of $\Sigma$. For the causality, now we replace $\mathbf{P}_{1}$ with $\mathbf{W}$. $\mathbf{Q}_{1}$ and $\mathbf{R}_{1}$ are obtained from the $\mathrm{QR}$ decomposition of $\Sigma \mathbf{W}$ as

$$
\mathbf{\Sigma W}=\mathbf{Q}_{1} \mathbf{R}_{1},
$$

where $\mathbf{Q}_{1}$ is also a $K \times K$ unitary matrix and $\mathbf{R}_{1}$ is a $K \times$ $K$ upper triangular matrix. Without loss of generality, we can make the diagonal entries of $\mathbf{R}_{1}$ positive and real. The detection algorithm at the receiver is exactly the same as in the ST-GTD transceiver. We call this kind of transceiver the causal ST-GTD transceiver (CST-GTD). It is in fact a subclass of ST-GTD. The error covariance matrix of the noise in front of detector is given by (11). And the total mean square error $\xi_{c s t-g t d}$ is the same as (12).

Now, we compare the performance of the conventional GMD-based system [6], the ST-GMD transceiver, and the CST-GTD transceiver. The total MSE of the GMD-based system in one ST-block is

$$
\xi_{g m d}=\frac{\sigma_{w}^{2}}{\alpha^{2}} M \sum_{k=0}^{K-1} \frac{1}{\sigma_{k}^{2}} .
$$

Theorem 2: The total mean square errors over one ST-block for the three transceivers are such that

$$
\xi_{s t-g m d} \leq \xi_{s t-g t d} \leq \xi_{g m d} .
$$

Proof. The first inequality follows from (12). To prove the second inequality, we firstly define a function

$$
f(\mathbf{x})=\frac{\sigma_{w}^{2} M}{\alpha^{2}} \sum_{k=0}^{K-1} e^{-2 x_{k}},
$$

where $f(\cdot): \mathbb{R}^{K} \mapsto \mathbb{R}$ and $\mathbf{x}=\left[x_{0}, x_{1}, \ldots, x_{K-1}\right]^{T}$. Since $e^{-2 x}, x \in \mathbb{R}$ is a convex function, $f(\mathbf{x})$ is a Schurconvex function by proposition 3.C.1 in [5]. Let $\Theta=$ $\left[\log \left|\eta_{0}\right|, \ldots, \log \left|\eta_{K-1}\right|\right]^{T}, \boldsymbol{\Gamma}=\left[\log \left|\sigma_{0}\right|, \ldots, \log \left|\sigma_{K-1}\right|\right]^{T}$. Since $\mathbf{r}_{1} \prec_{x} \mathbf{d}$, then $\boldsymbol{\Theta} \prec_{+} \boldsymbol{\Gamma}$. $\xi_{s t-g t d}$ in (12) and $\xi_{\text {gmd }}$ in (16) can be expressed in term of $\boldsymbol{\Gamma}$ and $\boldsymbol{\Theta}$ respectively 
as $\xi_{s t-g t d}=f(\boldsymbol{\Theta})$ and $\xi_{\text {gmd }}=f(\boldsymbol{\Gamma})$. Since $\boldsymbol{\Theta} \prec_{+} \boldsymbol{\Gamma}$, by the definition of Schur-convex function in [5], we have $\xi_{s t-g t d} \leq \xi_{\text {gmd }}$.

Therefore, we have proven that the class of ST-GTD transceivers has superior performance to the conventional GMD-based system in terms of arithmetic MSE for each STblock. In particular, (17) implies that $\xi_{s t-g m d} \leq \xi_{\text {cst-gtd }} \leq$ $\xi_{\text {gmd }}$. Also, note that the conventional GMD-based system is actually a subclass of CST-GTD with $\mathbf{W}=\mathbf{I}_{K}$.

\section{NUMERICAL RESULTS}

In this section, we will present the numerical results on the average BERs of the GMD-based system, ST-GMD and CST-GTD transceivers, denoted by $\mathcal{P}_{\text {gmd }}, \mathcal{P}_{s t-g m d}$ and $\mathcal{P}_{c s t-g t d}$, respectively. For square QAM, it is proven in [9] that $\mathcal{P}_{\text {gmd }} \leq \mathcal{P}_{\text {st-gmd }} \leq \mathcal{P}_{\text {cst-gtd }}$ in low SNR, and $\mathcal{P}_{s t-g m d} \leq \mathcal{P}_{c s t-g t d} \leq \mathcal{P}_{\text {gmd }}$ in high SNR.

We follow the channel model described in section 2. The noise is AWGN with power of $0 \mathrm{~dB}$. The MIMO channel matrices $\mathbf{H}(k)$ are $3 \times 3$ complex Gaussian random matrices. The elements of $\mathbf{H}(k)$ are i.i.d. complex Gaussian random variables with zero mean and unit variance. $\mathbf{H}(k)$ are independent for different $k$. Uniform bit allocation is adopted with $b=4$ for each substream. The modulation scheme is 16QAM. Both transmitter and receiver have perfect CSI at current time $k$. We assume that the channel prediction is available only for the ST-GMD transceiver. $2^{20}$ data blocks are sent through the channels for BER performance evaluation. The ST-block size is $K$. The temporal precoding matrix $\mathbf{W}$ of the CST-GTD is a $K \times K$ DFT matrix. Fig. 3 shows the BER performance of the GMD-based system, CST-GTD and ST-GMD transceivers for different SNR per symbol. For the entire SNR region, $\mathcal{P}_{s t-g m d}<\mathcal{P}_{c s t-g t d}$. The performance of CST-GTD is close to that of ST-GMD. At BER $10^{-5}$ and $K=32$, the SNR gain of the ST-GMD transceiver over the CST-GTD transceiver is only about $0.08 \mathrm{~dB}$. In the low SNR, $\mathcal{P}_{\text {st-gmd }}$ and $\mathcal{P}_{\text {cst-gtd }}$ are greater than $\mathcal{P}_{\text {gmd }}$. According to our simulations, the major factor at play is error propagation in the decision feedback loop. For the space-time processing at these receivers, the errors might propagate through the entire ST-block, i.e., $K$ blocks. In moderate high SNR, $\mathcal{P}_{\text {st-gmd }}<\mathcal{P}_{\text {cst-gtd }}<\mathcal{P}_{\text {gmd }}$. At BER $10^{-4}$ and $K=32$, the SNR gains of the ST-GMD and the CST-GTD over the GMD-based system are $5.15 \mathrm{~dB}, 5.03 \mathrm{~dB}$, respectively. It is also observed that increasing the ST-block size reduces the BER and also brings $\mathcal{P}_{s t-g m d}$ and $\mathcal{P}_{c s t-g t d}$ closer. These numerical results illustrate the great benefit of the time domain precoding in reasonably high SNR.

\section{CONCLUSIONS}

We have proposed two novel types of MIMO transceivers with zero-forcing decision feedback structure for MIMO time-varying channels. The case without zero-forcing constraint is under investigation. The proposed systems harvest the rich temporal diversity due to the time-varying nature of the channels to minimize the average BER. Under the assumption of perfect channel prediction, the ST-GMD transceiver is shown to be the best in terms of arithmetic MSE and average BER in high SNR. The CST-GTD has asymptotic BER performance similar to the ST-GMD transceiver and has smaller arithmetic MSE than the GMD-based systems.

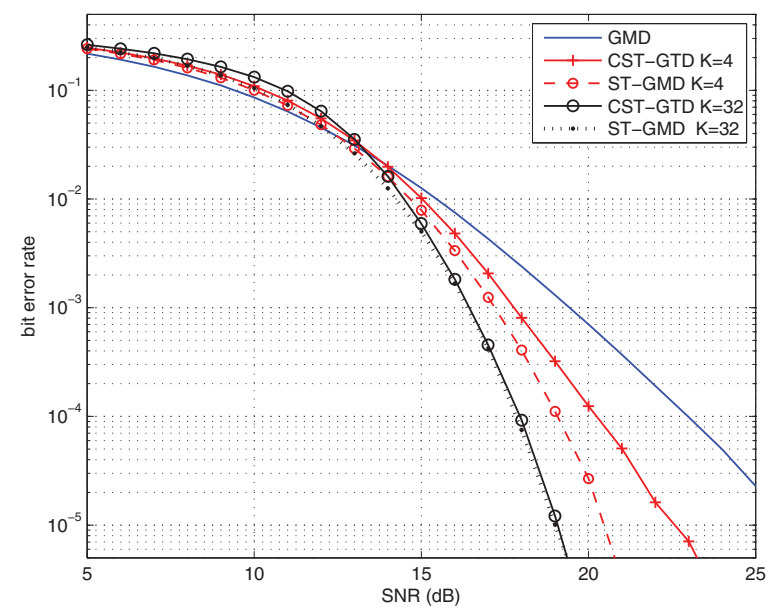

Fig. 3. BER performance of GMD, ST-GMD and CST-GTD.

\section{REFERENCES}

[1] A. Duel-Hallen, S. Hu, and H. Hallen, "Long-range prediction of fading signals," IEEE Signal Precessiong Mag., vol. 17, pp.62-75, May 2000.

[2] S. Zhou and G. B. Giannakis, "How accurate channel prediction needs to be for transmit-beamforming with adaptive modulation over Rayleigh MIMO channels?," IEEE Trans. Wireless Commun., vol. 3, no. 3, pp.1285-1294, July 2004.

[3] IEEE Std 802.16e, "Amendment for physical and medium access control layers for combined fixed and mobile operation in licensed bands," Sep. 2005.

[4] D. Tse and P. Viswanath, Fundamentals of Wireless Communication, Cambridge Univ. Press, 2005.

[5] A. W. Marshall and I. Olkin, Inequalities: Theory of majorization and its applications, Academic Press, 1979.

[6] Y. Jiang, J. Li, and W. W. Hager "Joint transceiver design for MIMO communications using geometric mean decomposition," IEEE Trans. Sig. Proc., pp.3791-3803, Oct. 2005.

[7] F. Xu, T. N. Davidson, J. K. Zhang and K. M. Wong, "Design of block transceivers with decision feedback detection," IEEE Trans. Sig. Proc., vol. 54, no. 3, pp. 965-978, Mar. 2006.

[8] Y. Jiang, W. W. Hager, and J. Li "Generalized triangular decomposition," Mathematics of computation., Oct. 2007.

[9] C. H. Liu and P. P. Vaidyanathan, "Transceiver design for MIMO time-varying channels using space-time generalized triangular decomposition," in preparation.

[10] Y. P. Lin and S. M. Phoong, "BER minimized OFDM systems with channel independent precoders," IEEE Trans. Sig. Proc., vol. 51, no. 9, pp.2369-2380, Sep. 2003.

[11] J. K. Zhang, A. Kavcic, and K. M. Wong, "Equaldiagonal QR decomposition and its application to precoder design for successive cancellation detection," IEEE Trans. Inf. Theory, vol. 51, no.1, pp.154-172, Jan. 2005. 